\title{
Weakly Orthomodular and Dually Weakly Orthomodular Lattices
}

\author{
Ivan Chajda ${ }^{1} \cdot$ Helmut Länger ${ }^{1,2}$
}

Received: 7 June 2017 / Accepted: 18 December 2017 / Published online: 20 January 2018

(C) The Author(s) 2018. This article is an open access publication

\begin{abstract}
We introduce so-called weakly orthomodular and dually weakly orthomodular lattices which are lattices with a unary operation satisfying formally the orthomodular law or its dual although neither boundedness nor complementation is assumed. It turns out that lattices being both weakly orthomodular and dually weakly orthomodular are in fact complemented but the complementation need not be neither antitone nor an involution. Moreover, every modular lattice with complementation is both weakly orthomodular and dually weakly orthomodular. The class of weakly orthomodular lattices and the class of dually weakly orthomodular lattices form varieties which are arithmetical and congruence regular. Connections to left residuated lattices are presented and commuting elements are introduced. Using commuting elements, we define a center of such a (dually) weakly orthomodular lattice and we provide conditions under which such lattices can be represented as a non-trivial direct product.
\end{abstract}

Keywords Weakly orthomodular lattice $\cdot$ Orthomodular lattice $\cdot$ Lattice with complementation $\cdot$ Residuated lattice $\cdot$ Congruence permutability $\cdot$ Congruence regularity

Helmut Länger

helmut.laenger@tuwien.ac.at

Ivan Chajda

ivan.chajda@upol.cz

1 Faculty of Science, Department of Algebra and Geometry, Palacký University Olomouc, 17. listopadu 12, 77146 Olomouc, Czech Republic

2 Faculty of Mathematics and Geoinformation, Institute of Discrete Mathematics and Geometry, TU Wien, Wiedner Hauptstraße 8-10, 1040 Vienna, Austria 


\section{Introduction}

Orthomodular lattices play an important role both in algebra and quantum mechanics. They were introduced independently by Birkhoff and von Neumann [3] and Husimi [9] as an algebraic semantic of the logic of quantum mechanics. Let us recall that an algebra ( $\left.L, \vee, \wedge,{ }^{\prime}, 0,1\right)$ of type $(2,2,1,0,0)$ is called an ortholattice if $(L, \vee, \wedge, 0,1)$ is a bounded lattice and 'satisfies the following conditions:

- $\quad x \vee x^{\prime} \approx 1$ and $x \wedge x^{\prime} \approx 0$ (' is a complementation),

- for all $x, y \in L, x \leq y$ implies $y^{\prime} \leq x^{\prime}$ (' is antitone),

- $\quad\left(x^{\prime}\right)^{\prime} \approx x\left(^{\prime}\right.$ is an involution).

Ortholattices were investigated in [1] and [2]. An ortholattice $\left(L, \vee, \wedge,{ }^{\prime}, 0,1\right)$ is called orthomodular if it satisfies one of the following equivalent conditions:

$$
\begin{aligned}
& x \leq y \text { implies } y=x \vee\left(y \wedge x^{\prime}\right), \\
& x \leq y \text { implies } x=y \wedge\left(x \vee y^{\prime}\right) .
\end{aligned}
$$

Since these conditions can be rewritten in the form of identities as follows

$$
\begin{aligned}
& x \approx(x \wedge y) \vee\left(x \wedge(x \wedge y)^{\prime}\right), \\
& x \approx(x \vee y) \wedge\left(x \vee(x \vee y)^{\prime}\right),
\end{aligned}
$$

the class of orthomodular lattices forms a variety. This variety is arithmetical, congruence regular and congruence uniform (cf. [1, 6] and [10]). Since ortholattices do not have these important properties except congruence distributivity, it is apparent that the strongness of orthomodular lattices is due to the orthomodular law (1), respectively (2). This motivated us to investigate algebras $\left(L, \vee, \wedge,,^{\prime}\right)$ such that $(L, \vee, \wedge)$ is a lattice and ' is a unary operation satisfying (1) or (2) or both.

It is our goal to show that the conditions (1) and/or (2) are so strong that we can prove a lot of results valid for orthomodular lattices (see e.g. [5, 10]) also in our more general setting. So one aim of the present paper is to demonstrate the strongness of the orthomodular law independently from the fact if the corresponding unary operation is antitone or an involution.

\section{Basic Properties}

Let $\mathbf{L}=(L, \vee, \wedge, 0,1)$ be a bounded lattice and ' a unary operation on $L$. We say that $\left(L, \vee, \wedge,{ }^{\prime}, 0,1\right)$ is a lattice with complementation if $x \vee x^{\prime} \approx 1$ and $x \wedge x^{\prime} \approx 0$. In this case ' is called a complementation. In general, we do not ask that the complementation' is either antitone or an involution. Hence, we cannot use the De Morgan laws in general.

Now, we show that conditions (1) and (2) are independent even in the case that the underlying lattice is a lattice with complementation. 
Since in the lattice with the following Hasse diagram (see e.g. [4])

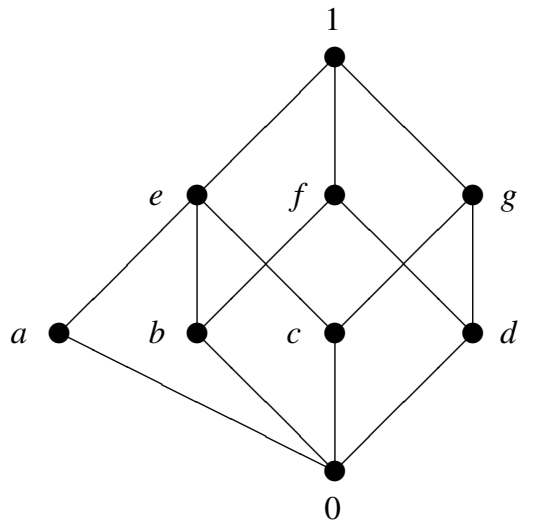

and the complementation defined by

$$
\begin{array}{l|lllllllll}
x & 0 & a & b & c & d & e & f & g & 1 \\
\hline x^{\prime} & 1 & g & g & f & e & d & c & b & 0
\end{array}
$$

we have

$$
\begin{aligned}
& a \vee\left(e \wedge a^{\prime}\right)=a \vee(e \wedge g)=a \vee c=e \text { and } \\
& e \wedge\left(a \vee e^{\prime}\right)=e \wedge(a \vee d)=e \wedge 1=e \neq a,
\end{aligned}
$$

this lattice with complementation satisfies (1), but it does not satisfy (2). Because of duality we see that (1) and (2) are independent for lattices with complementation.

Definition 2.1 A weakly orthomodular lattice is a lattice with unary operation satisfying (1) and a dually weakly orthomodular lattice is a lattice with unary operation satisfying (2).

The following lemma shows an important class of weakly orthomodular lattices.

Lemma 2.2 Every modular lattice with complementation is both weakly orthomodular and dually weakly orthomodular.

Proof If $\left(L, \vee, \wedge,{ }^{\prime}, 0,1\right)$ is a modular lattice with complementation, $a, b \in L$ and $a \leq b$ then

$$
\begin{aligned}
& a \vee\left(b \wedge a^{\prime}\right)=a \vee\left(a^{\prime} \wedge b\right)=\left(a \vee a^{\prime}\right) \wedge b=1 \wedge b=b \text { and } \\
& b \wedge\left(a \vee b^{\prime}\right)=\left(a \vee b^{\prime}\right) \wedge b=a \vee\left(b^{\prime} \wedge b\right)=a \vee 0=a .
\end{aligned}
$$

We are now going to show examples of modular lattices with complementation being not orthomodular. In such a case the complemenation need not be antitone nor an involution. 
Example 2.3 The lattice with the Hasse diagram

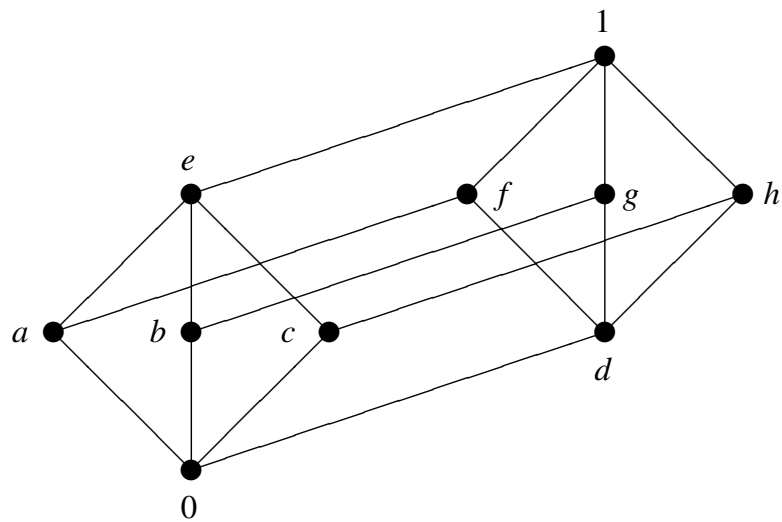

and the complementation defined by

$$
\begin{array}{l|llllllllll}
x & 0 & a & b & c & d & e & f & g & h & 1 \\
\hline x^{\prime} & 1 & g & h & f & e & d & c & a & b & 0
\end{array}
$$

is a modular lattice with complementation and hence both weakly orthomodular and dually weakly orthomodular and its complementation is an involution but not antitone since $a \leq f$, but $f^{\prime}=c \not \leq g=a^{\prime}$. The lattice with the Hasse diagram

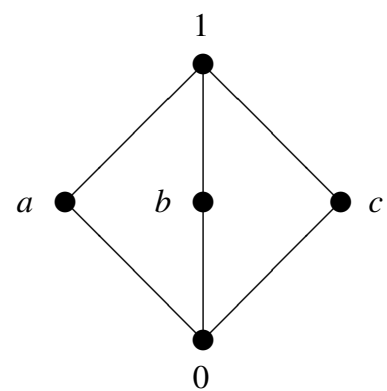

and the complementation defined by

$$
\begin{array}{l|lllll}
x & 0 & a & b & c & 1 \\
\hline x^{\prime} & 1 & b & c & a & 0
\end{array}
$$

is a modular lattice with complementation and hence both weakly orthomodular and dually weakly orthomodular and its complementation is antitone but not an involution since $\left(a^{\prime}\right)^{\prime}=$ $b^{\prime}=c \neq a$.

Now we show that conditions (1) and (2) are so strong that they already force a lattice $\left(L, \vee, \wedge,^{\prime}\right)$ with unary operation to be a bounded lattice with complementation.

Lemma 2.4 Every weakly orthomodular lattice $\mathbf{L}=\left(L, \vee, \wedge,,^{\prime}\right)$ has a greatest element 1 and satisfies $x \vee x^{\prime} \approx 1$. If $\mathbf{L}$ has, additionally, a smallest element 0 then $0^{\prime} \approx 1$. 
Proof For $a, b \in L$ we have

$$
a \leq a \vee b=b \vee\left((a \vee b) \wedge b^{\prime}\right) \leq b \vee b^{\prime}
$$

and

$$
a=0 \vee\left(a \wedge 0^{\prime}\right)=a \wedge 0^{\prime} \leq 0^{\prime}
$$

We obtain the following

Corollary 2.5 Every lattice $\mathbf{L}=\left(L, \vee, \wedge,{ }^{\prime}\right)$ being both weakly orthomodular and dually weakly orthomodular is bounded, ' is a complementation, $0^{\prime} \approx 1$ and $1^{\prime} \approx 0$ where 0 and 1 denote the smallest respectively greatest element of $\mathbf{L}$.

Moreover, conditions (1) and (2) remain valid in arbitrary intervals provided the complementation is defined suitably.

Lemma 2.6 If $\left(L, \vee, \wedge,{ }^{\prime}\right)$ is a weakly orthomodular lattice, $a \in L$ and $x_{a}:=x^{\prime} \wedge a$ and $x^{a}:=x^{\prime} \vee$ a for all $x \in L$ then $((a], \vee, \wedge, a)$ and $\left([a, 1], \vee, \wedge,{ }^{a}\right)$ are weakly orthomodular, too.

Proof If $b, c \in(a], b \leq c, d, e \in[a, 1]$ and $d \leq e$ then

$$
c=b \vee\left(c \wedge b^{\prime}\right)=b \vee\left(c \wedge\left(b^{\prime} \wedge a\right)\right)=b \vee\left(c \wedge b_{a}\right)
$$

and

$$
e=d \vee\left(e \wedge d^{\prime}\right) \leq d \vee\left(e \wedge\left(d^{\prime} \vee a\right)\right)=d \vee\left(e \wedge d^{a}\right) \leq e,
$$

i.e. $e=d \vee\left(e \wedge d^{a}\right)$.

Corollary 2.7 If $\mathbf{L}=\left(L, \vee, \wedge,,^{\prime}\right)$ is a weakly orthomodular lattice, $a, b \in L, a \leq b$ and $x^{*}:=\left(x^{\prime} \wedge b\right) \vee a$ and $x^{+}:=\left(x^{\prime} \vee a\right) \wedge b$ for all $x \in[a, b]$ then $\left([a, b], \vee, \wedge,{ }^{*}\right)$ and $\left([a, b], \vee, \wedge,{ }^{+}\right)$are weakly orthomodular, too. Because of duality, the same holds for dually weakly orthomodular lattices.

It should be remarked that if $\mathbf{L}$ is modular or orthomodular then ${ }^{*}$ and ${ }^{+}$coincide.

Contrary to the fact that every interval of a weakly orthomodular lattice is weakly orthomodular, that does not mean that certain properties of the corresponding complementation remain valid. For example, the complementation of the first weakly orthomodular lattice from Example 2.3 is an involution whereas the corresponding complementation in the interval $[d, 1]$ is not an involution as the following calculation shows:

$$
\left(g^{d}\right)^{d}=\left(g^{\prime} \vee d\right)^{\prime} \vee d=(a \vee d)^{\prime} \vee d=f^{\prime} \vee d=c \vee d=h \neq g .
$$

A lattice $\mathbf{L}=\left(L, \vee, \wedge,{ }^{\prime}\right)$ with unary operation satisfies Eqs. 1 and 2 if and only if it satisfies

$$
(x \wedge y) \vee\left(x \wedge(x \wedge y)^{\prime}\right) \approx(x \vee y) \wedge\left(x \vee(x \vee y)^{\prime}\right)
$$


This can be seen as follows: If $\mathbf{L}$ satisfies (1) and (2) then both sides of (3) are equal to $x$. If, conversely, $\mathbf{L}$ satisfies (3) then, because of

$$
(x \wedge y) \vee\left(x \wedge(x \wedge y)^{\prime}\right) \leq x \leq(x \vee y) \wedge\left(x \vee(x \vee y)^{\prime}\right)
$$

we obtain (1) and (2). Hence, the class of lattices being both weakly orthomodular and dually weakly orthomodular forms a variety. It is clear that such a lattice is orthomodular if and only if the De Morgan laws

$$
\begin{aligned}
& (x \vee y)^{\prime} \approx x^{\prime} \wedge y^{\prime} \text { and } \\
& (x \wedge y)^{\prime} \approx x^{\prime} \vee y^{\prime}
\end{aligned}
$$

and $\left(x^{\prime}\right)^{\prime} \approx x$ hold.

In what follows we describe a construction of lattices being both weakly orthomodular and dually weakly orthomodular which need not be modular. For this, we recall the concept of a horizontal sum.

Definition 2.8 Let $\mathbf{L}_{i}=\left(L_{i}, \vee_{i}, \wedge_{i},{ }^{\prime}, 0,1\right), i \in I$, be a non-empty family of non-trivial bounded lattices with a unary operation ${ }^{\prime}$ satisfying $0_{i}^{\prime} \approx 1$ and $1^{\prime}{ }_{i} \approx 0$. Moreover, assume $L_{i} \cap L_{j}=\{0,1\}$ for all $i, j \in I$ with $i \neq j$ and put $L:=\bigcup_{i \in I} L_{i}$. Define binary operations $\vee$ and $\wedge$ and a unary operation ' on $L$ as follows:

$$
\begin{aligned}
& x \vee y:= \begin{cases}x \vee_{j} y & \text { if }(x, y) \in L_{j}^{2} \\
1 & \text { if }(x, y) \in L^{2} \backslash\left(\bigcup_{i \in I} L_{i}^{2}\right),\end{cases} \\
& x \wedge y:=\left\{\begin{array}{ll}
x \wedge_{j} y & \text { if }(x, y) \in L_{j}^{2} \\
0 & \text { if }(x, y) \in L^{2} \backslash\left(\bigcup_{i \in I} L_{i}^{2}\right)
\end{array}\right. \text { and } \\
& x^{\prime}:=x^{\prime} \text { if } x \in L_{j}
\end{aligned}
$$

$(j \in I)$. Then $\left(L, \vee, \wedge,{ }^{\prime}, 0,1\right)$ is called the horizontal sum of the bounded lattices $\mathbf{L}_{i}, i \in I$.

It is easy to see that the horizontal sum of lattices being both weakly orthomodular and dually weakly orthomodular is again a lattice of this type. The horizontal sum of the lattices from Example 2.3 is a non-modular lattice being both weakly orthomodular and dually weakly orthomodular whose complementation is neither antitone nor an involution.

Let $\mathbf{P}=(P, \leq, 0)$ be a poset with smallest element 0 . Recall that the neighbors of 0 are called atoms, $\mathbf{P}$ is called atomic if every element of $P \backslash\{0\}$ lies over some atom and $\mathbf{P}$ is called atomistic if every element of $P$ is a join of atoms.

For weakly orthomodular lattices with complementation we can prove the following important result whose proof is adopted from that for orthomodular lattices.

Theorem 2.9 Every atomic weakly orthomodular lattice with complementation is atomistic.

Proof Let $\mathbf{L}=\left(L, \vee, \wedge,{ }^{\prime}, 0,1\right)$ be an atomic lattice with complementation satisfying (1), $a, b \in L$ and $A$ denote the set of all atoms $x$ of $\mathbf{L}$ with $x \leq a$ and assume $x \leq b$ for all $x \in A$. Suppose, $a \wedge b<a$. Because of $a=(a \wedge b) \vee\left(a \wedge(a \wedge b)^{\prime}\right)$ we have $a \wedge(a \wedge b)^{\prime} \neq 0$. Since $\mathbf{L}$ is atomic there exists an atom $c$ of $\mathbf{L}$ with $c \leq a \wedge(a \wedge b)^{\prime}$. This shows $c \leq a$, i.e. 
$c \in A$ and therefore $c \leq b$. We conclude $c \leq a \wedge b$ which together with $c \leq(a \wedge b)^{\prime}$ yields the contradiction $c \leq(a \wedge b) \wedge(a \wedge b)^{\prime}=0$. Therefore $a \wedge b=a$ which implies $a \leq b$. This shows $a=\bigvee A$.

\section{Commuting Elements}

It is well known that commuting elements play an important role in orthomodular lattices. A similar concept can be introduced also for weakly orthomodular lattices but with a slight modification caused by the fact that the De Morgan laws cannot be used here. Hence, we define

Definition 3.1 Let $\mathbf{L}=\left(L, \vee, \wedge,^{\prime}\right)$ be a lattice with unary operation and $a, b \in L$. We define a binary relation $\mathrm{C}$ on $L$ by $a \mathrm{C} b$ if and only if both

$$
\begin{aligned}
& (a \wedge b) \vee\left(a \wedge b^{\prime}\right)=a \\
& (a \vee b) \wedge\left(a \vee b^{\prime}\right)=a
\end{aligned}
$$

In this case we say that a commutes with $b$. It should be noticed that if $\mathbf{L}$ is orthomodular then (4) and (5) are equivalent. For every subset $M$ of $L$ we put $\mathbb{C}(M):=\{x \in L \mid$ $y \mathrm{C} x$ for all $y \in M$ \} and call $\mathbb{C}(L)$ the center of $\mathbf{L}$.

In every lattice being both weakly orthomodular and dually weakly orthomodular we have for arbitrary elements $x$ and $y: 0 \mathrm{C} x, 1 \mathrm{C} x, x \mathrm{C} 0, x \mathrm{C} 1, x \mathrm{C} x$ and $x^{\prime} \mathrm{C} x$. Moreover, if $x \leq y$ then $x \mathrm{C} y$ and $y \mathrm{C} x$.

Lemma 3.2 Let $\left(L, \vee, \wedge,{ }^{\prime}\right)$ be a lattice with unary operation and $a, b \in L$. Then the following hold:

(i) If $a \mathrm{C} b$ then

$$
\begin{aligned}
& a \vee b=b \vee\left(a \wedge b^{\prime}\right) \text { and } \\
& a \wedge b=b \wedge\left(a \vee b^{\prime}\right) .
\end{aligned}
$$

(ii) If $a \mathrm{C} b$ and $b \mathrm{C}$ a then

$$
\begin{aligned}
& \left(a \vee b^{\prime}\right) \wedge\left(a^{\prime} \vee b\right)=\left((a \wedge b) \vee a^{\prime}\right) \wedge\left((a \wedge b) \vee b^{\prime}\right) \text { and } \\
& \left(a \wedge b^{\prime}\right) \vee\left(a^{\prime} \wedge b\right)=\left((a \vee b) \wedge a^{\prime}\right) \vee\left((a \vee b) \wedge b^{\prime}\right) .
\end{aligned}
$$

Proof

(i) Since

$$
\begin{aligned}
& a=(a \wedge b) \vee\left(a \wedge b^{\prime}\right) \text { and } \\
& a=(a \vee b) \wedge\left(a \vee b^{\prime}\right)
\end{aligned}
$$

we have

$$
\begin{aligned}
& a \vee b=(a \wedge b) \vee\left(a \wedge b^{\prime}\right) \vee b=b \vee\left(a \wedge b^{\prime}\right) \text { and } \\
& a \wedge b=(a \vee b) \wedge\left(a \vee b^{\prime}\right) \wedge b=b \wedge\left(a \vee b^{\prime}\right) .
\end{aligned}
$$


(ii) Since

$$
\begin{aligned}
& a=(a \wedge b) \vee\left(a \wedge b^{\prime}\right), \\
& b=(b \wedge a) \vee\left(b \wedge a^{\prime}\right), \\
& a=(a \vee b) \wedge\left(a \vee b^{\prime}\right) \text { and } \\
& b=(b \vee a) \wedge\left(b \vee a^{\prime}\right)
\end{aligned}
$$

we have

$$
\begin{aligned}
\left(a \vee b^{\prime}\right) \wedge\left(a^{\prime} \vee b\right) & =\left((a \wedge b) \vee\left(a \wedge b^{\prime}\right) \vee b^{\prime}\right) \wedge\left(a^{\prime} \vee(b \wedge a) \vee\left(b \wedge a^{\prime}\right)\right) \\
& =\left((a \wedge b) \vee a^{\prime}\right) \wedge\left((a \wedge b) \vee b^{\prime}\right) \text { and } \\
\left(a \wedge b^{\prime}\right) \vee\left(a^{\prime} \wedge b\right) & =\left((a \vee b) \wedge\left(a \vee b^{\prime}\right) \wedge b^{\prime}\right) \vee\left(a^{\prime} \wedge(b \vee a) \wedge\left(b \vee a^{\prime}\right)\right) \\
& =\left((a \vee b) \wedge a^{\prime}\right) \vee\left((a \vee b) \wedge b^{\prime}\right)
\end{aligned}
$$

One can easily see that if $\mathbf{L}_{1}=\left(L_{1}, \vee, \wedge, 0,1\right), \mathbf{L}_{2}=\left(L_{2}, \vee, \wedge, 0,1\right)$ are lattices being both weakly orthomodular and dually weakly orthomodular, $\mathbf{L}:=\mathbf{L}_{1} \times \mathbf{L}_{2}$ and $a \in\{(0,1),(1,0)\}$ then $a \in \mathbb{C}(L)$ and $(x \wedge a)^{\prime}=x^{\prime} \vee a^{\prime}$ and $\left(x \wedge a^{\prime}\right)^{\prime}=x^{\prime} \vee a$ for all $x \in L$. Hence, these elements can be used for directly decomposing $\mathbf{L}$ as shown in the following theorem. Apparently, they satisfy rather strong properties.

Theorem 3.3 If $\mathbf{L}=\left(L, \vee, \wedge,{ }^{\prime}\right)$ is a dually weakly orthomodular lattice, 0 denotes its least element and $a \in \mathbb{C}(L)$ satisfies $(x \wedge a)^{\prime}=x^{\prime} \vee a^{\prime}$ and $\left(x \wedge a^{\prime}\right)^{\prime}=x^{\prime} \vee$ a for all $x \in L$ then

$$
\mathbf{L} \cong([0, a], \vee, \wedge, a, 0) \times\left(\left[0, a^{\prime}\right], \vee, \wedge, a^{\prime}, 0\right) .
$$

where $x_{a}:=x^{\prime} \wedge a$ and $x_{a^{\prime}}:=x^{\prime} \wedge a^{\prime}$ for all $x \in L$.

Proof Let $a \in \mathbb{C}(L)$ satisfy the mentioned assumptions, $b \in L,(c, d) \in[0, a] \times\left[0, a^{\prime}\right]$, $f$ denote the mapping $x \mapsto\left(x \wedge a, x \wedge a^{\prime}\right)$ from $L$ to $[0, a] \times\left[0, a^{\prime}\right]$ and $g$ the mapping $(x, y) \mapsto x \vee y$ from $[0, a] \times\left[0, a^{\prime}\right]$ to $L$. Then $g(f(b))=(b \wedge a) \vee\left(b \wedge a^{\prime}\right)=b$. Moreover, $c \leq(c \vee d) \wedge a \leq\left(c \vee a^{\prime}\right) \wedge a=c$ and

$$
d \leq(c \vee d) \wedge a^{\prime} \leq(a \vee d) \wedge a^{\prime}=(a \vee d) \wedge\left(a^{\prime} \vee d\right) \wedge a^{\prime}=d \wedge a^{\prime}=d,
$$

i.e. $f(g(c, d))=\left((c \vee d) \wedge a,(c \vee d) \wedge a^{\prime}\right)=(c, d)$. Hence $f$ and $g$ are mutually inverse bijections between $L$ and $[0, a] \times\left[0, a^{\prime}\right]$. Since they are obviously order-preserving, they are order isomorphisms and therefore lattice isomorphisms. Finally, we have

$$
\begin{aligned}
f\left(b^{\prime}\right) & =\left(b^{\prime} \wedge a, b^{\prime} \wedge a^{\prime}\right)=\left(\left(b^{\prime} \vee a\right) \wedge\left(b^{\prime} \vee a^{\prime}\right) \wedge a,\left(b^{\prime} \vee a\right) \wedge\left(b^{\prime} \vee a^{\prime}\right) \wedge a^{\prime}\right) \\
& =\left(\left(b^{\prime} \vee a^{\prime}\right) \wedge a,\left(b^{\prime} \vee a\right) \wedge a^{\prime}\right)=\left((b \wedge a)^{\prime} \wedge a,\left(b \wedge a^{\prime}\right)^{\prime} \wedge a^{\prime}\right) \\
& =\left((b \wedge a)_{a},\left(b \wedge a^{\prime}\right)_{a^{\prime}}\right)=\left(b \wedge a, b \wedge a^{\prime}\right)^{\prime}=(f(b))^{\prime}
\end{aligned}
$$

and $f(0)=(0,0)$. The equality $\left((b \wedge a)_{a},\left(b \wedge a^{\prime}\right)_{a^{\prime}}\right)=\left(b \wedge a, b \wedge a^{\prime}\right)^{\prime}$ follows from the definition of the unary operation within the direct product. 
Example 3.4 The lattice $\mathbf{L}$ with the Hasse diagram

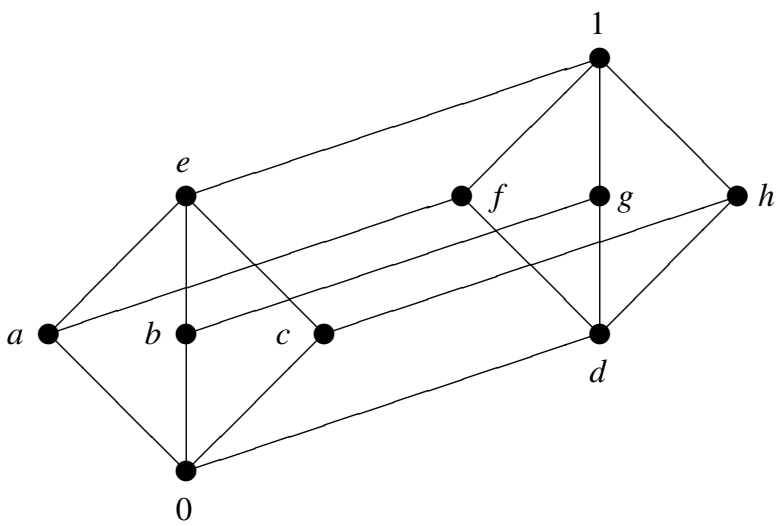

and the complementation defined by

$$
\begin{array}{l|llllllllll}
x & 0 & a & b & c & d & e & f & g & h & 1 \\
\hline x^{\prime} & 1 & g & h & f & e & d & b & c & a & 0
\end{array}
$$

is a modular lattice with complementation and hence dually weakly orthomodular. The element $e$ satisfies all assumptions of Theorem 3.3, $e^{\prime}=d$ and hence $\mathbf{L} \cong[0, e] \times[0, d]$. In fact, $[0, e]$ is the five-element weakly orthomodular lattice considered in Example 2.3 and $[0, d]$ a two-element weakly orthomodular lattice.

The lattice from Example 3.4 coincides with that of Example 2.3, but the complementation is defined in a different way. One can easily check that the element $e$ in Example 2.3 does not satisfy the assumptions of Theorem 3.3 and hence this theorem cannot be applied to the dually weakly orthomodular lattice of Example 2.3 .

In the following, for a subset $M$ of a lattice, $\langle M\rangle$ denotes the sublattice generated by $M$.

The well-known Foulis-Holland-Theorem for orthomodular lattices says that if an element of an orthomodular lattice commutes with two other elements then the lattice generated by all three elements is distributive. Using Theorem 3.3 we obtain a similar result for dually weakly orthomodular lattices.

Theorem 3.5 If $\mathbf{L}=\left(L, \vee, \wedge,^{\prime}\right)$ is a dually weakly orthomodular lattice, 0 denotes its least element, $a \in \mathbb{C}(L),(x \wedge a)^{\prime}=x^{\prime} \vee a^{\prime}$ and $\left(x \wedge a^{\prime}\right)^{\prime}=x^{\prime} \vee$ a for all $x \in L$, and $b, c \in L$ then $\langle\{a, b, c\}\rangle$ is distributive.

Proof From the proof of Theorem 3.3 we have that $x \mapsto\left(x \wedge a, x \wedge a^{\prime}\right)$ is an isomorphism from $(L, \vee, \wedge)$ to $([0, a], \vee, \wedge) \times\left(\left[0, a^{\prime}\right], \vee, \wedge\right)$. Now

$$
\langle\{a, b, c\}\rangle \cong\left\langle\left\{(a, 0),\left(b \wedge a, b \wedge a^{\prime}\right),\left(c \wedge a, c \wedge a^{\prime}\right)\right\}\right\rangle
$$

which is a sublattice of

$$
\begin{aligned}
\langle\{a, b \wedge a, c \wedge a\}\rangle \times\left\langle\left\{0, b \wedge a^{\prime}, c \wedge a^{\prime}\right\}\right\rangle= & \{a, b \wedge a, c \wedge a, b \wedge c \wedge a,(b \wedge a) \vee(c \wedge a)\} \\
& \times\left\{0, b \wedge a^{\prime}, c \wedge a^{\prime}, b \wedge c \wedge a^{\prime},\left(b \wedge a^{\prime}\right) \vee\left(c \wedge a^{\prime}\right)\right\} .
\end{aligned}
$$


Since the last two lattices have at most one pair of incomparable elements, they cannot contain $M_{3}$ nor $N_{5}$ as a sublattice and hence they are distributive. This shows that also $\langle\{a, b, c\}\rangle$ is distributive.

Example 3.6 Since the element $e$ of Example 3.4 satisfies the assumption of Theorem 3.5 we have that $\langle\{e, x, y\}\rangle$ is distributive for all elements $x, y$ of this lattice.

The following theorem generalizes the corresponding theorem for orthomodular lattices. The proof follows the same lines as that of the corresponding theorem for orthomodular lattices.

Theorem 3.7 If $\mathbf{L}=\left(L, \vee, \wedge,{ }^{\prime}\right)$ is a dually weakly orthomodular lattice, $M \subseteq L, a \in$ $\mathbb{C}(M)$ and $\bigvee M$ exists then also $\bigvee_{m \in M}(m \wedge a)$ exists and $\bigvee_{m \in M}(m \wedge a)=(\bigvee M) \wedge a$.

Proof Obviously, $m \wedge a \leq(\bigvee M) \wedge a$ for all $m \in M$. Let $b \in L$ and assume $m \wedge a \leq b$ for all $m \in M$. Then

$m \leq m \vee a^{\prime}=(m \wedge a) \vee\left(m \wedge a^{\prime}\right) \vee a^{\prime}=(m \wedge a) \vee a^{\prime}=(m \wedge a \wedge b) \vee a^{\prime} \leq((\bigvee M) \wedge a \wedge b) \vee a^{\prime}$

for all $m \in M$. Hence $\bigvee M \leq((\bigvee M) \wedge a \wedge b) \vee a^{\prime}$ and therefore

$$
a \wedge(\bigvee M) \leq a \wedge\left(((\bigvee M) \wedge a \wedge b) \vee a^{\prime}\right)=(\bigvee M) \wedge a \wedge b \leq b
$$

This shows $(\bigvee M) \wedge a=\bigvee_{m \in M}(m \wedge a)$.

\section{Left Residuated Lattices}

Orthomodular lattices form an algebraic semantic for the logic of quantum mechanics (see e.g. [3] and [9]). In particular, MV-algebras are residuated lattices which form an algebraic semantic for many-valued Łukasiewicz logics; moreover, divisible and prelinear residuated lattices form a semantic for Hajek's BL-logics etc. All of these logics are so-called substructural logics. We have already developed several attempts to connect various semantics, see e.g. [7] and [8]. Hence, the question arises if a similar approach is possible for lattices being both weakly orthomodular and dually weakly orthomodular. At first, we define the main concept of this section.

Definition 4.1 A left residuated lattice is an algebra $\mathbf{R}=(R, \vee, \wedge, \odot, \rightarrow, 0,1)$ of type $(2,2,2,2,0,0)$ such that $(R, \vee, \wedge, 0,1)$ is a bounded lattice, 1 is the neutral element of $(R, \odot)$ and the left adjointness property holds: For all $x, y, z \in R$,

$$
x \odot y \leq z \text { is equivalent to } x \leq y \rightarrow z .
$$

The algebra $\mathbf{R}$ is called idempotent if the identity $x \odot x \approx x$ holds.

A connection between lattices being both weakly orthomodular and dually weakly orthomodular with left residuated lattices can be established if the operations $\odot$ and $\rightarrow$ are defined as shown in the following theorem: 
Theorem 4.2 If $\mathbf{L}=\left(L, \vee, \wedge,{ }^{\prime}, 0,1\right)$ is a lattice being both weakly orthomodular and dually weakly orthomodular and satisfying the identity $\left(x^{\prime}\right)^{\prime} \approx x$ and one defines

$$
\begin{aligned}
x \odot y & :=\left(x \vee y^{\prime}\right) \wedge y \text { and } \\
x \rightarrow y & :=x^{\prime} \vee(x \wedge y)
\end{aligned}
$$

for all $x, y \in L$ then $(L, \vee, \wedge, \odot, \rightarrow, 0,1)$ is an idempotent left residuated lattice satisfying the identity $x \odot(x \vee y) \approx x$.

Proof Let $a, b, c \in L$. Then we have

$$
\begin{aligned}
a \odot a & =\left(a \vee a^{\prime}\right) \wedge a=1 \wedge a=a, \\
a \odot 1 & =\left(a \vee 1^{\prime}\right) \wedge 1=(a \vee 0) \wedge 1=a \wedge 1=a, \\
1 \odot a & =\left(1 \vee a^{\prime}\right) \wedge a=1 \wedge a=a \text { and } \\
a \odot(a \vee b) & =\left(a \vee(a \vee b)^{\prime}\right) \wedge(a \vee b)=a .
\end{aligned}
$$

Moreover, if $a \odot b \leq c$ then

$a \leq a \vee b^{\prime}=b^{\prime} \vee\left(\left(a \vee b^{\prime}\right) \wedge b\right)=b^{\prime} \vee\left(b \wedge\left(\left(a \vee b^{\prime}\right) \wedge b\right)\right)=b^{\prime} \vee(b \wedge(a \odot b)) \leq b^{\prime} \vee(b \wedge c)=b \rightarrow c$

Finally, if $a \leq b \rightarrow c$ then

$a \odot b=\left(a \vee b^{\prime}\right) \wedge b \leq\left((b \rightarrow c) \vee b^{\prime}\right) \wedge b=\left(\left(b^{\prime} \vee(b \wedge c)\right) \vee b^{\prime}\right) \wedge b=\left(b^{\prime} \vee(b \wedge c)\right) \wedge b=b \wedge c \leq c$

If the left residuated lattice is modular, we can prove the converse.

Theorem 4.3 If $\mathbf{R}=(R, \vee, \wedge, \odot, \rightarrow, 0,1)$ is a modular left residuated lattice satisfying the identities

$$
\begin{aligned}
x \odot y & \approx\left(x \vee y^{\prime}\right) \wedge y \text { and } \\
x \rightarrow y & \approx x^{\prime} \vee(x \wedge y)
\end{aligned}
$$

where $x^{\prime}:=x \rightarrow 0$ for all $x \in R$ then $\left(R, \vee, \wedge,{ }^{\prime}, 0,1\right)$ is a lattice being both weakly orthomodular and dually weakly orthomodular.

Proof If $a \in R$ then $1 \leq a \rightarrow 1=a^{\prime} \vee(a \wedge 1)=a \vee a^{\prime}$ since $1 \odot a \leq 1$ and $a \wedge a^{\prime}=\left(0 \vee a^{\prime}\right) \wedge a=0 \odot a \leq 0$ since $0 \leq a \rightarrow 0$. This shows that ${ }^{\prime}$ is a complementation. According to Lemma 2.2 we are done.

If modularity is not assumed we must ask for stronger assumptions.

Theorem 4.4 If $\mathbf{R}=(R, \vee, \wedge, \odot, \rightarrow, 0,1)$ is a left residuated lattice satisfying the identities

$$
\begin{aligned}
x \odot y & \approx\left(x \vee y^{\prime}\right) \wedge y, \\
x^{\prime} \rightarrow y & \approx x \vee\left(x^{\prime} \wedge y\right), \\
x \odot(x \vee y) & \approx x \text { and } \\
x^{\prime} \rightarrow(x \vee y) & \approx x \vee y
\end{aligned}
$$


where $x^{\prime}:=x \rightarrow 0$ for all $x \in R$ then $\left(R, \vee, \wedge,{ }^{\prime}, 0,1\right)$ is a lattice being both weakly orthomodular and dually weakly orthomodular.

Proof If $a, b \in R$ and $a \leq b$ then

$$
\begin{aligned}
& b=a \vee b=a^{\prime} \rightarrow(a \vee b)=a^{\prime} \rightarrow b=a \vee\left(b \wedge a^{\prime}\right) \text { and } \\
& a=a \odot(a \vee b)=a \odot b=b \wedge\left(a \vee b^{\prime}\right) .
\end{aligned}
$$

\section{Congruence Properties}

Congruence properties form an important tool for revealing essential features of algebras. In particular, this is true for varieties of algebras. It is well-known (see e.g. [1] and [10]) that the variety of orthomodular lattices is arithmetical and congruence regular. In what follows we show that the same is true also for the variety of weakly orthomodular lattices. For the reader's convenience, we firstly recall these properties.

Definition 5.1 A variety $\mathcal{V}$ is called

- $\quad$ permutable if $\Theta \circ \Phi=\Phi \circ \Theta$ for all $\mathbf{A} \in \mathcal{V}$ and $\Theta, \Phi \in \operatorname{Con} \mathbf{A}$,

- distributive if $(\Theta \vee \Phi) \cap \Psi=(\Theta \cap \Psi) \vee(\Phi \cap \Psi)$ for all $\mathbf{A} \in \mathcal{V}$ and $\Theta, \Phi, \Psi \in$ Con $\mathbf{A}$,

- arithmetical if it is both permutable and distributive and

- congruence regular if for all $\mathbf{A}=(A, F) \in \mathcal{V}, a \in A$ and $\Theta, \Phi \in \operatorname{Con} \mathbf{A},[a] \Theta=[a] \Phi$ implies $\Theta=\Phi$.

For the following proposition, see e.g. the monograph [6].

Proposition 5.2 Let $\mathcal{V}$ be a variety.

- $\mathcal{V}$ is permutable if and only if there exists a ternary term $p$ satisfying $p(x, x, y) \approx$ $p(y, x, x) \approx y$.

- If there exists a ternary term $m$ satisfying $m(x, x, y) \approx m(x, y, x) \approx m(y, x, x) \approx x$ then $\mathcal{V}$ is distributive.

- $\quad \mathcal{V}$ is congruence regular if and only if there exists a positive integer $n$ and ternary terms $t_{1}, \ldots, t_{n}$ such that $t_{1}(x, y, z)=\cdots=t_{n}(x, y, z)=z$ is equivalent to $x=y$.

Now we can state the main result of this section.

Theorem 5.3 The variety of weakly orthomodular lattices with complementation is arithmetical and congruence regular. According to duality, the same is true for the variety of dually weakly orthomodular lattices with complementation.

Proof If

$$
\begin{aligned}
p(x, y, z) & :=\left(x \vee\left((z \vee y) \wedge y^{\prime}\right)\right) \wedge\left(z \vee\left((x \vee y) \wedge y^{\prime}\right)\right), \\
m(x, y, z) & :=(x \vee y) \wedge(y \vee z) \wedge(z \vee x), \\
t_{1}(x, y, z) & :=\left((x \vee y) \wedge(x \wedge y)^{\prime}\right) \vee z \text { and } \\
t_{2}(x, y, z): & \left((x \vee y) \wedge(x \wedge y)^{\prime}\right)^{\prime} \wedge z
\end{aligned}
$$


then

$$
\begin{aligned}
& p(x, x, y) \approx\left(x \vee\left((y \vee x) \wedge x^{\prime}\right)\right) \wedge\left(y \vee\left((x \vee x) \wedge x^{\prime}\right)\right) \approx(x \vee y) \wedge y \approx y, \\
& p(y, x, x) \approx\left(y \vee\left((x \vee x) \wedge x^{\prime}\right)\right) \wedge\left(x \vee\left((y \vee x) \wedge x^{\prime}\right)\right) \approx y \wedge(x \vee y) \approx y, \\
& m(x, x, y) \approx(x \vee x) \wedge(x \vee y) \wedge(y \vee x) \approx x \wedge(x \vee y) \approx x, \\
& m(x, y, x) \approx(x \vee y) \wedge(y \vee x) \wedge(x \vee x) \approx(x \vee y) \wedge x \approx x, \\
& m(y, x, x) \approx(y \vee x) \wedge(x \vee x) \wedge(x \vee y) \approx(x \vee y) \wedge x \approx x, \\
& t_{1}(x, x, z) \approx\left((x \vee x) \wedge(x \wedge x)^{\prime}\right) \vee z \approx z, \\
& t_{2}(x, x, z) \approx\left((x \vee x) \wedge(x \wedge x)^{\prime}\right)^{\prime} \wedge z \approx z
\end{aligned}
$$

and $t_{1}(x, y, z)=t_{2}(x . y, z)=z$ implies $u=u \wedge(u \vee z)=u \wedge\left(u^{\prime} \wedge z\right)=0$ where $u:=(x \vee y) \wedge(x \wedge y)^{\prime}$ and hence

$$
x=x \wedge(x \vee y)=x \wedge((x \wedge y) \vee u)=x \wedge y=y \wedge((x \wedge y) \vee u)=y \wedge(x \vee y)=y .
$$

According to Theorem 5.3, every weakly orthomdular lattice with complementation is congruence regular and thus each of its congruences is determined by any single class. This dependence is described in the following

Proposition 5.4 Let $\mathbf{L}=\left(L, \vee, \wedge,{ }^{\prime}, 0,1\right)$ be a weakly orthomodular lattice with complementation, $a, b, c \in L$ and $\Theta \in \mathbf{C o n} \mathbf{L}$. Then the following are equivalent:

(i) $(a, b) \in \Theta$

(ii) $\quad\left((a \vee b) \wedge(a \wedge b)^{\prime}\right) \vee c,\left((a \vee b) \wedge(a \wedge b)^{\prime}\right)^{\prime} \wedge c \in[c] \Theta$

(iii) $(a \vee b) \wedge(a \wedge b)^{\prime} \in[0] \Theta$

(iv) $\left((a \vee b) \wedge(a \wedge b)^{\prime}\right)^{\prime} \in[1] \Theta$

Proof Put $d:=(a \vee b) \wedge(a \wedge b)^{\prime}$.

(i) $\Rightarrow$ (ii):

We have

$$
\begin{aligned}
& d \vee c \Theta\left((a \vee a) \wedge(a \wedge a)^{\prime}\right) \vee c=c \text { and } \\
& d^{\prime} \wedge c \Theta\left((a \vee a) \wedge(a \wedge a)^{\prime}\right)^{\prime} \wedge c=c .
\end{aligned}
$$

(ii) $\Rightarrow$ (i):

We have $d=d \wedge(d \vee c) \Theta d \wedge\left(d^{\prime} \wedge c\right)=0$ and hence

$$
a=a \wedge(a \vee b)=a \wedge((a \wedge b) \vee d) \Theta a \wedge b \Theta b \wedge((a \wedge b) \vee d)=b \wedge(a \vee b)=b .
$$

That (iii) and (iv) are equivalent to (i) follows from the equivalence of (i) and (ii) in case $c=0$ respectively in case $c=1$.

Proposition 5.5 Let $\mathbf{L}=\left(L, \vee, \wedge,{ }^{\prime}, 0,1\right)$ be a modular lattice with complementation, $a, b \in L$ and $\Theta \in$ Con $\mathbf{L}$. Then the following are equivalent:

(i) $(a, b) \in \Theta$

(ii) $(a \vee b) \wedge\left(a^{\prime} \vee b^{\prime}\right) \in[0] \Theta$

(iii) There exists some $c \in[0] \Theta$ with $a \vee c=b \vee c$. 
Proof

(i) $\Rightarrow$ (ii):

We have $(a \vee b) \wedge\left(a^{\prime} \vee b^{\prime}\right) \Theta(a \vee a) \wedge\left(a^{\prime} \vee a^{\prime}\right)=0$

(ii) $\Rightarrow$ (iii):

Using modularity and the facts that $a \leq a \vee b$ and $b \leq a \vee b$ we have

$$
\begin{aligned}
a \vee\left((a \vee b) \wedge\left(a^{\prime} \vee b^{\prime}\right)\right) & =\left(a \vee\left(a^{\prime} \vee b^{\prime}\right)\right) \wedge(a \vee b)=a \vee b=\left(b \vee\left(a^{\prime} \vee b^{\prime}\right)\right) \wedge(a \vee b) \\
& =b \vee\left((a \vee b) \wedge\left(a^{\prime} \vee b^{\prime}\right)\right) .
\end{aligned}
$$

(iii) $\Rightarrow$ (i):

We have $a=a \vee 0 \Theta a \vee c=b \vee c \Theta b \vee 0=b$.

Definition 5.6 Let $\mathbf{L}=\left(L, \vee, \wedge,^{\prime}\right)$ be a lattice with unary operation. An ideal $I$ of $(L, \vee, \wedge)$ is called a $w$-ideal of $\mathbf{L}$ if for all $x, y, z \in L$ the following hold:

(i) $(x \vee y) \wedge\left(x^{\prime} \vee y^{\prime}\right) \in I$ if and only if there exists an $u \in I$ with $x \vee u=y \vee u$.

(ii) If $(x \vee y) \wedge\left(x^{\prime} \vee y^{\prime}\right) \in I$ then $((x \wedge z) \vee(y \wedge z)) \wedge\left((x \wedge z)^{\prime} \vee(y \wedge z)^{\prime}\right) \in I$.

Let w-Id $\mathbf{L}$ denote the set of all w-ideals of $\mathbf{L}$.

Definition 5.7 For any join-semilattice $(L, \vee)$ and any subset $M$ of $L$ put

$$
\Theta_{M}:=\left\{(x, y) \in L^{2} \mid \text { there exists some } z \in M \text { with } x \vee z=y \vee z\right\} .
$$

Theorem 5.8 Let $\mathbf{L}=\left(L, \vee, \wedge,{ }^{\prime}, 0,1\right)$ be a modular lattice with complementation which is an involution. Then $\Theta \mapsto[0] \Theta$ and $I \mapsto \Theta_{I}$ are mutually inverse isomorphisms between $(\mathrm{Con} \mathbf{L}, \subseteq)$ and $(\mathrm{w}-\mathrm{Id} \mathbf{L}, \subseteq)$.

Proof Let $a, b, c \in L, \Phi, \Psi \in \operatorname{Con} \mathbf{L}$ and $I, J \in \mathrm{w}-\operatorname{Id} \mathbf{L}$. Then [0] $\Phi$ is a lattice ideal of $\mathbf{L}$ and according to Proposition 5.5, [0] $\Phi \in \mathrm{w}$-Id $\mathbf{L}$. Since $I \neq \emptyset, \Theta_{I}$ is reflexive. Obviously, $\Theta_{I}$ is symmetric. If $(a, b),(b, c) \in \Theta_{I}$ then there exist $d, e \in I$ with $a \vee d=b \vee d$ and $b \vee e=c \vee e$ and hence

$$
a \vee(d \vee e)=(a \vee d) \vee e=(b \vee d) \vee e=(b \vee e) \vee d=(c \vee e) \vee d=c \vee(d \vee e)
$$

i.e. $(a, c) \in \Theta_{I}$ since $d \vee e \in I$. This shows that $\Theta_{I}$ is transitive and therefore an equivalence relation on $L$. That $\Theta_{I}$ has the substitution property with respect to $\vee$ follows immediately from the definition of $\Theta_{I}$. Because of (i) of Definition 5.6 and the assumption that ' is an involution, $\Theta_{I}$ has the substitution property with respect to ' . According to Proposition 5.5 and (ii) of Definition 5.6, $\Theta_{I}$ has the substitution property with respect to $\wedge$. This shows $\Theta_{I} \in$ Con $\mathbf{L}$. According to Proposition 5.5, $\Theta_{[0] \Phi}=\Phi$ and since

$$
a \in[0] \Theta_{I} ; \text { there exists some } d \in I \text { with } a \vee d=0 \vee d ; a \in I
$$

are equivalent, we have $[0] \Theta_{I}=I$. Finally, $\Phi \subseteq \Psi$ implies $[0] \Phi \subseteq[0] \Psi$, and $I \subseteq J$ implies $\Theta_{I} \subseteq \Theta_{J}$ completing the proof of the theorem.

Example 5.9 One can easily check that $I:=\{0, a, b, c, e\}$ is a non-trivial w-ideal of the modular lattice with complementation which is an involution from Example 2.3. 
The corresponding congruence is $\{0, a, b, c, e\}^{2} \cup\{d, f, g, h, 1\}^{2}$. For example, for the elements $d$ and $f$ we have

$$
(d \vee f) \wedge\left(d^{\prime} \vee f^{\prime}\right)=f \wedge(e \vee c)=f \wedge e=a \in I
$$

and one can check that $d \vee a=f=f \vee a$ and $a \in I$ in accordance with condition (i) of Definition 5.6.

Acknowledgments Open access funding provided by Austrian Science Fund (FWF). Support of the research of both authors by ÖAD, project CZ 04/2017, as well as by IGA, project PřF 2018 012, is gratefully acknowledged. Support of the second author by the Austrian Science Fund (FWF), project I 1923-25 entitled "New perspectives on residuated posets", is gratefully acknowledged.

Open Access This article is distributed under the terms of the Creative Commons Attribution 4.0 International License (http://creativecommons.org/licenses/by/4.0/), which permits unrestricted use, distribution, and reproduction in any medium, provided you give appropriate credit to the original author(s) and the source, provide a link to the Creative Commons license, and indicate if changes were made.

\section{References}

1. Beran, L.: Orthomodular Lattices. Algebraic Approach. Reidel, Dordrecht (1985). ISBN 90-277-1715-X

2. Birkhoff, G.: Lattice Theory. AMS, Providence (1979). ISBN 0-8218-1025-1

3. Birkhoff, G., von Neumann, J.: The logic of quantum mechanics. Ann. of Math. 37, 823-843 (1936)

4. Bonzio, S., Chajda, I.: A note on orthomodular lattices. Internat. J. Theoret. Phys. 56, 3740-3743 (2017)

5. Bruns, G., Harding, J.: Algebraic aspects of orthomodular lattices. Fund. Theories Phys. 111, 37-65 (2000). Kluwer, Dordrecht

6. Chajda, I., Eigenthaler, G., Länger, H.: Congruence Classes in Universal Algebra. Heldermann, Lemgo (2012). ISBN 3-88538-226-1

7. Chajda, I., Länger, H.: Residuation in orthomodular lattices. Topological Algebra Appl. 5, 1-5 (2017)

8. Chajda, I., Länger, H.: Orthomodular lattices can be converted into left residuated l-groupoids. Miskolc Math. Notes (to appear)

9. Husimi, K.: Studies of the foundation of quantum mechanics. I. Proc. Phys.-Math. Soc. Japan 19, 766789 (1937)

10. Kalmbach, G.: Orthomodular Lattices. Academic Press, London (1983). ISBN 0-12-394580-1 